\title{
A high tibial slope, allograft use, and poor patient-reported outcome scores are associated with multiple ACL graft failures
}

\author{
Philipp W. Winkler ${ }^{1,2}$ (D) Nyaluma N. Wagala ${ }^{1} \cdot$ Jonathan D. Hughes ${ }^{1} \cdot$ Bryson P. Lesniak $^{1} \cdot$ Volker Musahl $^{1}$
}

Received: 23 November 2020 / Accepted: 18 January 2021 / Published online: 31 January 2021

(c) The Author(s) 2021

\begin{abstract}
Purpose To compare clinical outcomes, radiographic characteristics, and surgical factors between patients with single and multiple anterior cruciate ligament (ACL) graft failures. It was hypothesized that patients experiencing multiple ACL graft failures exhibit lower patient-reported outcome scores (PROs) and a higher (steeper) posterior tibial slope (PTS) than patients with single ACL graft failure.

Methods Patients undergoing revision ACL reconstruction with a minimum follow-up of 12 months were included in this retrospective cohort study. Based on the number of ACL graft failures, patients were assigned either to the group "single ACL graft failure "or" multiple ACL graft failures ". The PTS was measured on strict lateral radiographs. Validated PROs including the International Knee Documentation Committee (IKDC) subjective knee form, Knee Injury and Osteoarthritis Outcome Score, Lysholm Score, Tegner Activity Scale, ACL-Return to Sport after Injury Scale, and Visual Analogue Scale for pain were collected.

Results Overall, 102 patients were included with 58 patients assigned to the single ACL graft failure group and 44 patients to the multiple ACL graft failures group. Quadriceps tendon autograft was used significantly more often (55\% vs. $11 \%$, $p<0.001)$ and allografts were used significantly less often $(31 \%$ vs. $66 \%, p<0.001)$ as the graft for first revision ACL reconstruction in patients with single versus multiple ACL graft failures. Patients with multiple ACL graft failures were associated with statistically significantly worse PROs (IKDC: $61.7 \pm 19.3$ vs. $77.4 \pm 16.8, p<0.05$; Tegner Activity Scale: 4 (range, $0-7)$ vs. 6 (range $2-10), p<0.05)$, higher PTS $\left(12 \pm 3^{\circ}\right.$ vs. $\left.9 \pm 3^{\circ}, p<0.001\right)$, and higher rates of subsequent surgery $(73 \%$ vs. $14 \%, p<0.001)$ and complications $(45 \%$ vs. $17 \%, p<0.05)$ than patients with single ACL graft failure.

Conclusion Compared to single ACL graft failure in this study multiple ACL graft failures were associated with worse PROs, higher PTS, and allograft use. During the first revision ACL reconstruction, it is recommended to avoid the use of allografts and to consider slope-reducing osteotomies to avoid multiple ACL graft failures and improve PROs.
\end{abstract}

Level of evidence Level 3.

Keywords ACL · Anterior cruciate ligament $\cdot$ Failure $\cdot$ Revision $\cdot$ Quadriceps tendon $\cdot$ Allograft $\cdot$ Tibial slope

\section{Abbreviations}

ACL Anterior cruciate ligament

ACL-R Anterior cruciate ligament reconstruction

BMI Body mass index

ICC Intraclass correlation coefficient

Volker Musahl

musahlv@upmc.edu

1 Department of Orthopaedic Surgery, UPMC Freddie Fu Sports Medicine Center, University of Pittsburgh, 3200 S. Water St, Pittsburgh, PA 15203, USA

2 Department for Orthopaedic Sports Medicine, Klinikum rechts der Isar, Technical University of Munich, Ismaninger Str. 22, 81675 Munich, Germany
LFCR Lateral femoral condyle ratio

MARS Multicenter anterior cruciate ligament revision study

MRI Magnetic resonance imaging

OA Osteoarthritis

PROs Patient-reported outcomes

PTS Posterior tibial slope

\section{Introduction}

The failure rate after revision anterior cruciate ligament reconstruction (ACL-R) has been reported to be $3-21 \%$ [1-5] compared to $3-10 \%$ after primary ACL-R [6-11]. 
Anatomical and patient-related risk factors, technical and biological failures, unappreciated concomitant capsuloligamentous or meniscal injuries, as well as aggravated surgical conditions due to previous interventions have been identified as underlying causes for the increased failure rate [1, 12-15].

Despite a large body of the literature regarding single anterior cruciate ligament (ACL) graft failures, only a few studies emphasizing multiple ACL graft failures have been published $[1,5,12,13,15,16]$. Prior studies have shown that undergoing more than one revision ACL-R is a predictor for worse patient-reported outcomes (PROs) and subsequent graft failures [2,17]. Additionally, the majority of patients that have sustained multiple ACL graft failures present with meniscal tears and cartilage lesions $[5,12,13,15$, 16], which are well-known negative predictors for long-term knee function. Failure analyses revealed that an increased posterior tibial slope (PTS), static anterior tibial subluxation, and a deep, elliptically shaped, lateral femoral condyle were associated with multiple ACL graft failures [1, 5, 13]. Consequently, a failure rate of up to $30 \%$ after a second revision ACL-R was demonstrated [5]. Additionally, it has been shown that patients undergoing more than two revision ACL-Rs are almost 26 times more likely to experience subsequent ACL graft failure than patients undergoing first revision ACL-R [2]. Thus, a vicious circle of multiple ACL graft failures appears to exist in certain patients. Further investigation of the multifactorial etiology of ACL graft failures is warranted to avoid the vicious circle of multiple ACL graft failures. Surgery- and patient-related predictors of multiple ACL graft failures may aid in surgical decision-making and patient counseling for revision ACL reconstruction.

The objectives of this study were to compare clinical outcomes, demographic and radiographic characteristics, and surgical factors between patients with single and multiple ACL graft failures. It was hypothesized that patients experiencing multiple ACL graft failures exhibit lower PROs and a higher (steeper) PTS than patients with single ACL graft failure.

\section{Materials and methods}

This study was approved by the Institutional Review Board of the University of Pittsburgh (No.: STUDY20050226). All patients presenting with single or multiple ACL graft failures in the senior author's (VM) outpatient clinic between 2010 and 2020 were screened for eligibility for this retrospective cohort study.

Inclusion criteria comprised: single-bundle first revision ACL-R, minimum 12 month follow-up since the first revision ACL-R, available medical records, and anterior-posterior (weight-bearing) and lateral radiographs. Patients with a history of inflammatory arthritis, ipsilateral multiple-ligament knee injuries, or a previous femur or tibia fracture were excluded from this study. Given the need for revision ACL-R to sustain multiple ACL graft failures, the first revision ACL-R was considered as the index operation (Fig. 1). Therefore, patients undergoing non-operative treatment after the first ACL graft failure were excluded from this study.

Anterior cruciate ligament graft failure was defined as (1) the need for revision ACL-R due to symptomatic instability, pain, or severe impairment in daily activities, (2) complete ACL graft disruption confirmed by magnetic resonance imaging (MRI) or arthroscopy, or (3) attenuated or partially ruptured graft confirmed by MRI plus side-to-side difference $>5 \mathrm{~mm}$ for anterior tibial translation based on KT-1000

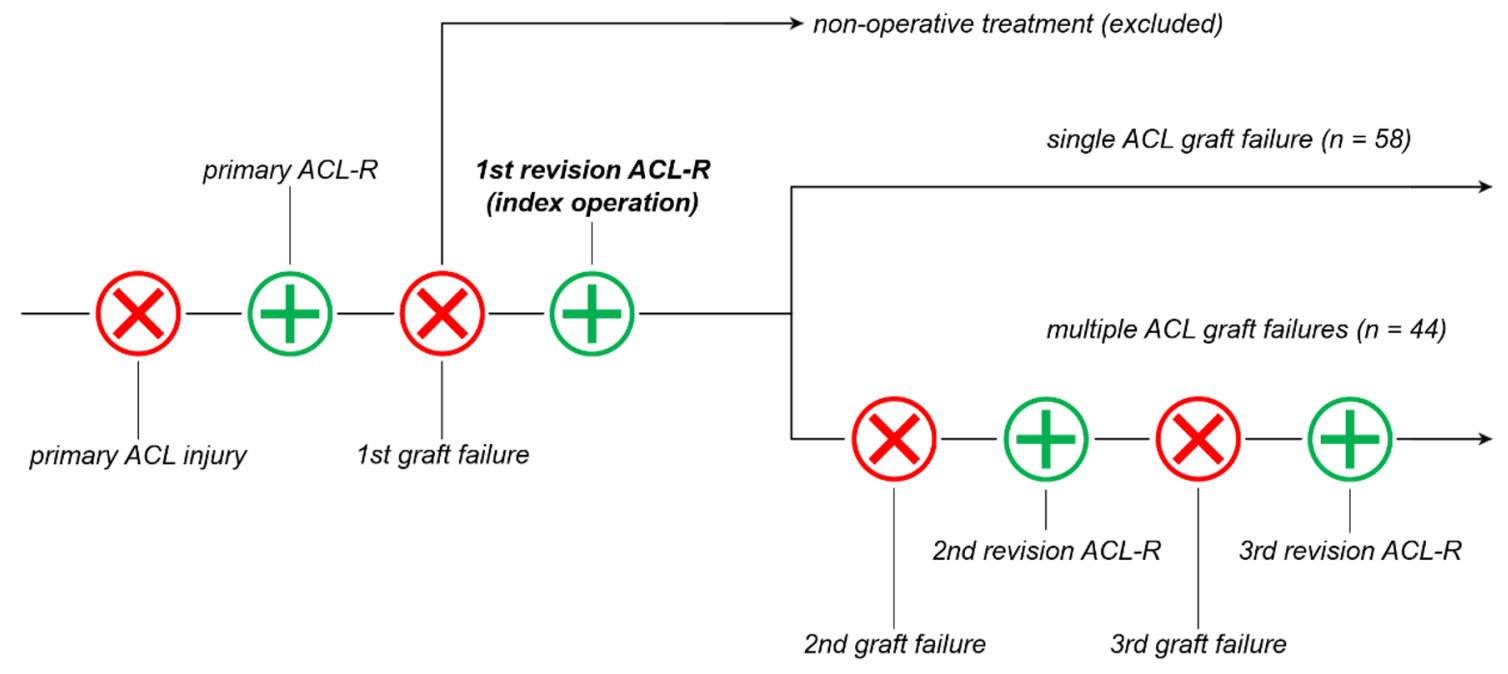

Fig. 1 Group allocation based on the number of anterior cruciate ligament graft failures. Encircled " $x$ " illustrating ACL injury. Encircled "+" illustrating ACL reconstruction. ACL, anterior cruciate ligament; ACL-R, ACL reconstruction 
(MEDmetric Corp., San Diego, CA, USA) arthrometry. Based on the number of ACL graft failures, the patients were subsequently assigned either to the group "single ACL graft failure" or "multiple ACL graft failures" (Fig. 2).

\section{Data collection}

\section{Demographic and surgical data}

A comprehensive review of medical records was conducted between March and July 2020 by one observer (PWW). Demographic and surgical data were collected and included the following: sex; laterality; body mass index (BMI); date of birth; date of each ACL injury; date of each ACL-R performed; mechanism of each ACL injury; occurrence, number, and type of complications; occurrence, number, and type of subsequent surgical procedures; concurrently performed surgical procedures during first revision ACL$\mathrm{R}$; graft choices; and history of contralateral ACL injuries. Atraumatic, traumatic non-contact, and traumatic contact were defined as the three types of injury mechanisms. Complications were categorized and included the following: symptomatic hardware, surgical site infection, meniscus tear requiring reoperation, cartilage lesion requiring reoperation, knee stiffness $\left(10^{\circ}\right.$ side-to-side difference in range of motion based on clinical examination), and symptomatic baker cyst confirmed by MRI.

\section{Radiographic characteristics}

Lateral radiographs with a maximum posterior femoral condyle overlap of $6 \mathrm{~mm}$ were used to measure the medial PTS as well as the lateral femoral condyle ratio (LFCR), as previously described in detail [18-21]. Briefly, the medial PTS was defined as the angle between the proximal tibial shaft axis and a tangential line to the medial tibial plateau, subtracted from $90^{\circ}[18,21]$. To calculate the LFCR, first the distal femoral shaft axis was determined on lateral radiographs. In a second step, the maximum anterior to posterior distance of the lateral femoral condyle (lateral femoral condyle axis) was measured. Next, the distance along the lateral femoral condyle axis between the most posterior part of the lateral condyle and the femoral shaft axis was measured (posterior lateral femoral condyle depth). Subsequently, the

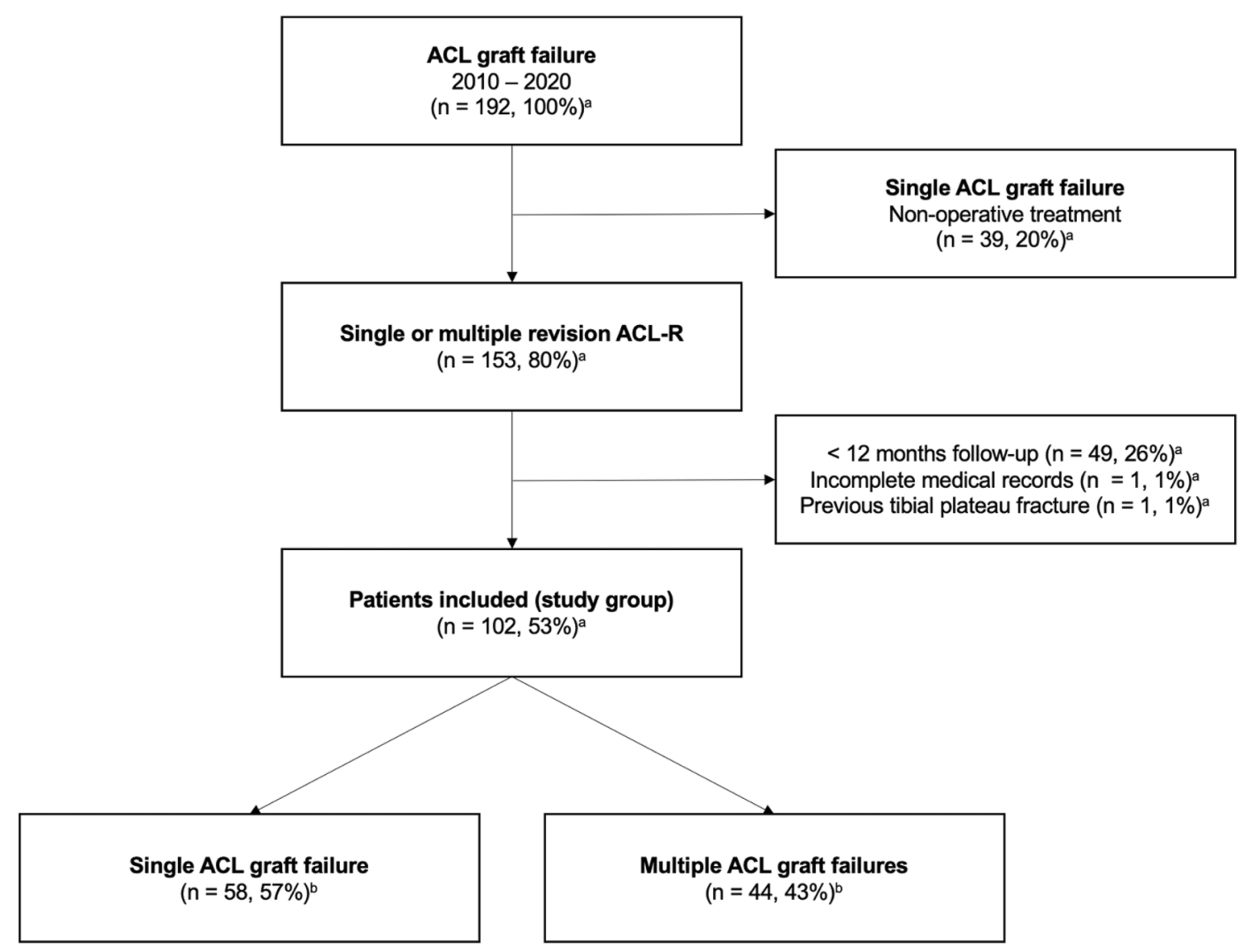

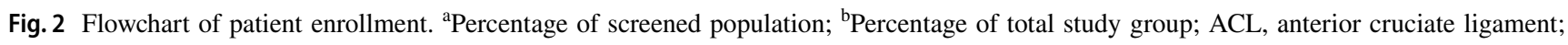
ACL-R, ACL reconstruction 
posterior lateral femoral condyle depth was divided by the total anterior-posterior length of the lateral femoral condyle to obtain the LFCR $[19,20]$. All measurements were performed by observer one (PWW) using Philips iSite PACS (Koninklijke Philips N.V., Amsterdam, NLD), which allows a measurement accuracy of $0.1 \mathrm{~mm}$ and $1^{\circ}$, respectively. Intraclass correlation coefficients (ICC) were calculated to assess intra- and interrater reliability for the measurements obtained. Accordingly, the medial PTS and LFCR were measured twice by observer one (PWW; 1-month interval between measurements) and once by observer 2 (NNW) for 20 randomly selected patients. Intrarater ICC of 0.817 (LFCR) and 0.853 (medial PTS) as well as interrater ICC of 0.846 (LFCR) and 0.825 (medial PTS) indicate good reliability of the measurements. Additionally, anterior-posterior weight-bearing radiographs were used to determine the degree of osteoarthritis (OA) based on the Kellgren-Lawrence Scale. The degree of OA was assessed by observers one (PWW) and two (NNW) in agreement with each other.

\section{Patient-reported outcome scores (PROs)}

Questionnaires including standardized and validated PROs were mailed to all included patients. The following PROs were used for follow-up assessment: International Knee Documentation Committee (IKDC) subjective knee form, Knee Injury and Osteoarthritis Outcome Score (KOOS), Lysholm Score, Tegner Activity Scale, ACL-Return to Sport after Injury Scale (ACL-RSI) [22], and Visual Analogue Scale (VAS) for pain. Written informed consent was obtained from each patient who completed the questionnaire.

\section{Statistical analysis}

A priori power analysis was conducted using the freely available software G*Power (Erdfelder, Faul, Buchner, Lang, HHU Düsseldorf, Düsseldorf, Germany). The PTS is known as a risk factor for ACL graft failure. Therefore, the PTS was considered to be the primary outcome measure and was used for a priori sample size calculation. A recently published study reported a mean PTS of $11.5^{\circ} \pm 3.6^{\circ}$ and $13.1^{\circ} \pm 2.4^{\circ}$ in patients undergoing revision ACL-R and rerevision ACL-R, respectively [5]. Accordingly, a total sample size of 92 patients (46 patients per group) was required to achieve a statistical power of 0.8 (effect size, 0.52 ; level of significance, 0.05 ).

Categorical variables are presented as count and percentage. Normal distribution of the collected continuous variables was assessed by the Shapiro-Wilk test. Accordingly, continuous variables are presented either as mean and standard deviation or as median and range, as appropriate. Group comparison of categorical variables was performed using the Chi-square test (followed by post-hoc testing with
Bonferroni corrected $p$ values) or the Fisher's exact test, as appropriate. The Fisher's exact test was used if the expected count of a cell was less than five. For group comparison of continuous variables, the Mann-Whitney $U$ test or the unpaired $t$ test was applied. SPSS software version 26.0 (IBM-SPSS, New York, USA) was used for statistical analysis and the level of significance was set at $p<0.05$.

\section{Results}

A total of 102 patients with a median age of 24 years (range 13-58 years) at the time of the index procedure (first revision ACL-R) were included in this study. The single ACL graft failure group consisted of 58 patients with a median follow-up since the index procedure of 29 months (range 12-124 months), while the multiple ACL graft failures group consisted of 44 patients with a median follow-up of 85 months (range 12-272 months). The follow-up time from the index procedure was statistically significantly longer for the multiple ACL graft failures group compared to the single ACL graft failure group $(p<0.001)$. A detailed summary of the demographic, surgical, and radiographic data is shown in Table 1.

Group comparison revealed that statistically significantly more patients underwent first revision ACL-R using quadriceps tendon autograft in the single ACL graft failure group compared to the multiple ACL graft failures group (32 (55\%) vs. $5(11 \%), p<0.001)$. Moreover, statistically significantly less allografts were used for first revision ACL-R in the single ACL graft failure group than in the multiple ACL graft failures group (18 (31\%) vs. $29(66 \%), p<0.001)$. With respect to radiographic characteristics, multiple ACL graft failures were associated with a statistically significantly higher (steeper) medial PTS than single ACL graft failures $\left(12 \pm 3^{\circ}\right.$ vs. $\left.9 \pm 3^{\circ}, p<0.001\right)$. Although only available for 42 patients (41\% of the study group) statistically significantly worse PROs were observed in patients with multiple ACL graft failures compared to patients with single ACL graft failures on all scores collected except for the KOOS subscale "activities of daily living" (Table 2, Fig. 3).

A total of 93 subsequent surgical procedures were performed after the index procedure. In the multiple ACL graft failures group, statistically significantly more patients underwent subsequent surgical procedures compared to the single ACL graft failure group (32 (73\%) vs. $8(14 \%), p<0.001)$. Similarly, significantly more patients had complications in the multiple ACL graft failures group than in the single ACL graft failure group $(20(45 \%)$ vs. $10(17 \%), p<0.05)$, resulting in a total of 37 complications. A detailed summary of the subsequent surgical procedures performed and the complications observed is shown in Table 3. 
Table 1 Demographic, surgical, and radiographic data

\begin{tabular}{|c|c|c|c|}
\hline Variable & Single ACL graft failure & Multiple ACL graft failures & $p$ value \\
\hline Number of patients, $n$ & 58 & 44 & - \\
\hline Age at first revision ACL-R, ${ }^{a}$ (years) & $22.5(13-58)$ & $24.0(15-49)$ & n.s \\
\hline Follow-up since first revision ACL-R, ${ }^{\mathrm{a}}$ (months) & $29.0(12-124)$ & $85.0(12-272)$ & $<0.001 *$ \\
\hline Age at primary ACL-R, ${ }^{\mathrm{a}}$ (years) & $17.5(12-53)$ & $17.0(12-34)$ & n.s \\
\hline Primary ACL-R to first graft failure, ${ }^{\mathrm{a}}$ (months) & $18.0(0-300)$ & $16.5(1-275)$ & n.s \\
\hline Primary ACL-R to first revision ACL-R, (months) ${ }^{a}$ & $23.0(4-308)$ & $26.5(2-279)$ & n.s \\
\hline BMI, $\left(\mathrm{kg} / \mathrm{m}^{2}\right)$ & $27.0 \pm 5.1(20.5-42.8)$ & $27.4 \pm 5.3(19.6-40.5)$ & n.s \\
\hline Males, $n(\%)$ & $29(50 \%)$ & $24(55 \%)$ & n.s \\
\hline Right knee, $n(\%)$ & $27(47 \%)$ & $15(34 \%)$ & n.s \\
\hline Graft primary ACL-R & & & n.s \\
\hline Hamstring tendon, $n(\%)$ & $21(36 \%)$ & $22(50 \%)$ & \\
\hline Quadriceps tendon, $n(\%)$ & $3(5 \%)$ & $1(2 \%)$ & \\
\hline Allograft, $n(\%)$ & $16(28 \%)$ & $10(23 \%)$ & \\
\hline $\mathrm{N} / \mathrm{A}, n(\%)$ & $1(2 \%)$ & $0(0 \%)$ & \\
\hline Graft first revision ACL-R & & & $<0.001 *$ \\
\hline Hamstring tendon, $n(\%)$ & $1(2 \%)$ & $4(9 \%)$ & \\
\hline Quadriceps tendon, $n(\%)$ & $32(55 \%)$ & $5(11 \%)$ & \\
\hline Bone-patellar tendon-bone, $n(\%)$ & $7(12 \%)$ & $6(14 \%)$ & \\
\hline Allograft, $n(\%)$ & $18(31 \%)$ & $29(66 \%)$ & \\
\hline Injury mechanism first graft failure & & & $<0.05 *$ \\
\hline Atraumatic, $n(\%)$ & $13(22 \%)$ & $20(45 \%)$ & \\
\hline Traumatic non-contact, $n(\%)$ & $41(71 \%)$ & $20(45 \%)$ & \\
\hline Traumatic contact, $n(\%)$ & $4(7 \%)$ & $4(9 \%)$ & \\
\hline Meniscus surgery at first revision ACL-R, $n(\%)$ & $49(84 \%)$ & $42(95 \%)$ & n.s \\
\hline Concomitant surgical procedure at first revision ACL-R & & & n.s \\
\hline None, $n(\%)$ & $44(76 \%)$ & $39(89 \%)$ & \\
\hline Meniscal allograft transplantation, $n(\%)$ & $1(2 \%)$ & $3(7 \%)$ & \\
\hline Lateral extra-articular tenodesis, $n(\%)$ & $6(10 \%)$ & $1(2 \%)$ & \\
\hline Osteotomy, $n(\%)$ & $2(3 \%)$ & $0(0 \%)$ & \\
\hline Cartilage surgery, $n(\%)$ & $5(9 \%)$ & $1(2 \%)$ & \\
\hline Contralateral ACL injury & $14(24 \%)$ & $6(14 \%)$ & n.s \\
\hline Kellgren-Lawrence Scale & & & n.s \\
\hline Grade $0, n(\%)$ & $21(36 \%)$ & $10(23 \%)$ & \\
\hline Grade $1, n(\%)$ & $18(31 \%)$ & $11(25 \%)$ & \\
\hline Grade $2, n(\%)$ & $14(24 \%)$ & $11(25 \%)$ & \\
\hline Grade $3, n(\%)$ & $5(9 \%)$ & $11(25 \%)$ & \\
\hline Grade $4, n(\%)$ & $0(0 \%)$ & $1(2 \%)$ & \\
\hline Lateral femoral condyle ratio, $(-)^{\mathrm{b}}$ & $0.65 \pm 0.04(0.56-0.73)$ & $0.65 \pm 0.04(0.60-0.75)$ & n.s \\
\hline Medial posterior tibial slope, $\left({ }^{\circ}\right)^{b}$ & $9 \pm 3(3-18)$ & $12 \pm 3(6-17)$ & $<0.001 *$ \\
\hline
\end{tabular}

Categorical variables are presented as count (percentage of the corresponding group). Continuous variables are presented as mean \pm standard deviation (range), unless otherwise noted

ACL, anterior cruciate ligament; ACL-R, anterior cruciate ligament reconstruction; BMI, body mass index; N/A, not available; n.s., non-significant

* Statistically significant difference $(p<0.05)$

${ }^{a}$ Median (range)

${ }^{\mathrm{b}}$ Data available for 98 patients (4 patients had to be excluded because of $>6 \mathrm{~mm}$ posterior femoral condyle overlap) 
Table 2 Patient-reported outcome scores

\begin{tabular}{llll}
\hline Variable $^{\mathrm{a}}$ & Single ACL graft failure & Multiple ACL graft failures & $p$ value \\
\hline IKDC & & & \\
KOOS & $77.4 \pm 16.8(32.2-100)$ & $61.7 \pm 19.3(17.2-95.4)$ & $<\mathbf{0 . 0 5}^{*}$ \\
Symptoms & $76.8 \pm 18.7(28.6-100)$ & $59.9 \pm 21.5(17.9-92.9)$ & $<\mathbf{0 . 0 5}^{*}$ \\
Pain & $91.8 \pm 10.0(55.6-100)$ & $75.2 \pm 22.4(11.1-100)$ & $<\mathbf{0 . 0 5}^{*}$ \\
ADL & $96.3 \pm 6.0(76.5-100)$ & $87.7 \pm 23.0(16.2-100)$ & n.s \\
Sport/Rec & $77.5 \pm 24.7(10.0-100)$ & $45.4 \pm 26.7(0-100)$ & $<\mathbf{0 . 0 5}^{*}$ \\
QOL & $63.0 \pm 26.5(0-100)$ & $32.2 \pm 27.7(0-93.8)$ & $<\mathbf{0 . 0 5}^{*}$ \\
Lysholm Score & $83.6 \pm 16.6(25.0-100)$ & $69.9 \pm 22.1(14.0-95.0)$ & $<\mathbf{0 . 0 5}^{*}$ \\
Tegner Activity Scale & $6(2-10)$ & $4(0-7)$ & $<\mathbf{0 . 0 5}^{*}$ \\
ACL-RSI & $45.1 \pm 28.6(0-99.1)$ & $11.0 \pm 18.8(0-66.7)$ & $<\mathbf{0 . 0 0 1}^{*}$ \\
VAS (pain) & $0.9 \pm 1.6(0-6)$ & $2.0 \pm 2.4(0-8)$ & $<\mathbf{0 . 0 5}^{*}$ \\
\hline
\end{tabular}

Continuous variables are presented as mean \pm standard deviation (range), unless otherwise noted

ACL, anterior cruciate ligament; ACL-RSI, ACL-Return to Sport after Injury Scale; ADL, activities of daily living; IKDC, International Knee Documentation Committee subjective knee form; KOOS, Knee Injury and Osteoarthritis Outcome Score; n.s., non-significant; Sport/Rec, sport and recreation function; QOL, knee-related quality of life; VAS, visual analogue scale

*Statistically significant difference $(p<0.05)$

${ }^{a}$ Data available for 42 patients ( $41 \%$ of study group)

${ }^{b}$ Median (range)

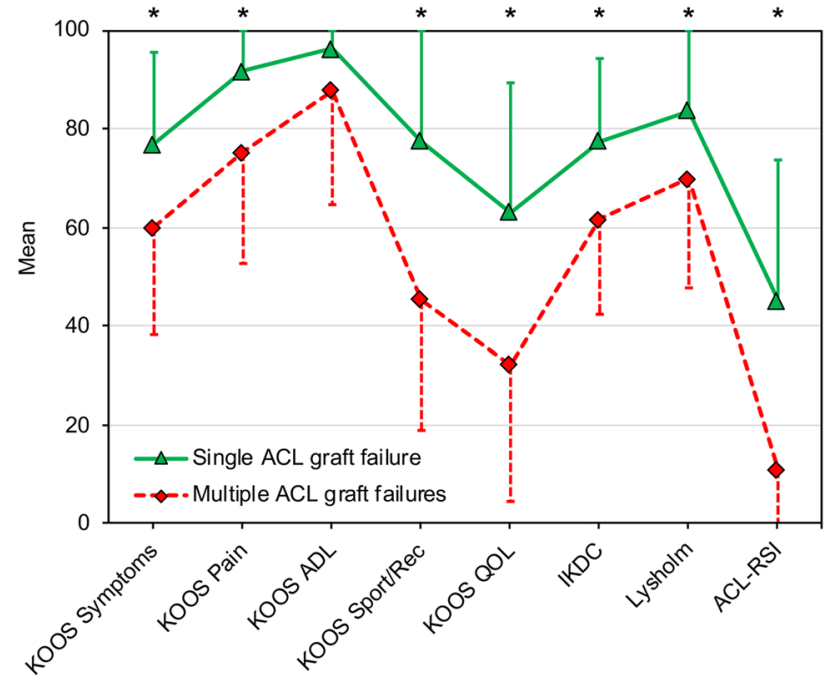

Fig. 3 Patient-reported outcome scores with a maximum score of 100 points. Indicators (triangle, rhombus) represent mean values. Error bars represent standard deviation. ACL-RSI, ACL-Return to Sport after Injury Scale; ADL, activities of daily living; IKDC, International Knee Documentation Committee subjective knee form; KOOS, Knee Injury and Osteoarthritis Outcome Score; Sport/Rec, sport and recreation function; QOL, knee-related quality of life; *, statistically significant difference $(p<0.05)$

\section{Discussion}

The most important finding of this study was that compared to patients with single ACL graft failure patients with multiple ACL graft failures were associated with worse PROs, higher medial PTS $\left(12^{\circ}\right.$ vs. $\left.9^{\circ}\right)$, less quadriceps tendon autograft use ( $11 \%$ vs. $55 \%)$, and more allograft use (66\% vs. $31 \%)$.

Similar to primary ACL-R, graft choices for revision ACL-R remain a highly debated topic subjected to many controversies [2-4]. The Multicenter ACL Revision Study (MARS) group has shown that the graft choice affects PROs and failure rates in revision ACL-R [2]. More precisely, patients undergoing revision ACL-R using autografts have been shown to be almost three times less likely to sustain a subsequent graft failure compared to patients undergoing allograft revision ACL-R [2]. Similar results have been shown by another study, which demonstrated a significantly higher Lysholm Score at a mean time of 53 months after revision ACL-R when autografts were used compared to allografts [4]. In addition, the failure rate after revision ACL-R was higher for allografts than for autografts ( $27 \%$ vs. $11 \%$ ), although not statistically significant [4]. Accordingly, it is evident that autografts are superior to allografts in revision ACL-R, but no final recommendation for the type of autograft has yet been made $[2,4]$. The present study showed that in patients with a single ACL graft failure, quadriceps tendon autografts were significantly more often used in first revision ACL-R than in patients with multiple ACL graft failures, where allografts were predominant. The versatility of the quadriceps tendon in ACL-R has been demonstrated in numerous studies and is further supported by the results of this study $[9,23,24]$. While the use of the quadriceps tendon, either with or without an attached patellar bone block, has been established and accepted as a viable graft option 
Table 3 Subsequent surgical procedures and complications

\begin{tabular}{|c|c|c|c|}
\hline Variable & Single ACL graft failure & $\begin{array}{l}\text { Multiple ACL } \\
\text { graft failures }\end{array}$ & $p$ value \\
\hline Subsequent surgical procedure, n (\%) & $8(14 \%)$ & $32(73 \%)$ & $<0.001 *$ \\
\hline $\begin{array}{l}\text { Number of subsequent surgical procedures } \\
\text { (per patient) }\end{array}$ & $1.1 \pm 0.4(1-2)$ & $2.0 \pm 1.2(1-7)$ & $<0.05 *$ \\
\hline Type of subsequent surgical procedures ${ }^{a}$ & & & $<0.001 *$ \\
\hline Revision ACL-R, $n(\%)$ & $0(0 \%)$ & $35(42 \%)$ & \\
\hline Hardware removal, $n(\%)$ & $2(22 \%)$ & $8(10 \%)$ & \\
\hline Irrigation/Debridement, $n(\%)$ & $4(44 \%)$ & $4(5 \%)$ & \\
\hline Manipulation under anesthesia, $n(\%)$ & $1(11 \%)$ & $2(2 \%)$ & \\
\hline Meniscus surgery, $n(\%)$ & $2(22 \%)$ & $3(4 \%)$ & \\
\hline Osteotomy, $n(\%)$ & $0(0 \%)$ & $9(11 \%)$ & \\
\hline Cartilage surgery, $n(\%)$ & $0(0 \%)$ & $6(7 \%)$ & \\
\hline Meniscal allograft transplantation, $n(\%)$ & $0(0 \%)$ & $13(15 \%)$ & \\
\hline Lateral extra-articular tenodesis, $n(\%)$ & $0(0 \%)$ & $4(5 \%)$ & \\
\hline Complication, $n(\%)$ & $10(17 \%)$ & $20(45 \%)$ & $<0.05 *$ \\
\hline Number of complications (per patient) & $1.1 \pm 0.3(1-2)$ & $1.3 \pm 0.5(1-2)$ & n.s \\
\hline Type of complications ${ }^{b}$ & & & n.s \\
\hline Symptomatic hardware, $n(\%)$ & $2(18 \%)$ & $8(31 \%)$ & \\
\hline Surgical site infection, n (\%) & $3(27 \%)$ & $2(8 \%)$ & \\
\hline Meniscus tear, $n(\%)$ & $4(36 \%)$ & $7(27 \%)$ & \\
\hline Cartilage lesion, $n(\%)$ & $0(0 \%)$ & $6(23 \%)$ & \\
\hline Knee stiffness, $n(\%)$ & $1(9 \%)$ & $3(12 \%)$ & \\
\hline Symptomatic baker cyst, $n(\%)$ & $1(9 \%)$ & $0(0 \%)$ & \\
\hline
\end{tabular}

Categorical variables are presented as count (percentage of the corresponding group). Continuous variables are presented as mean \pm standard deviation (range)

ACL, anterior cruciate ligament; ACL-R, anterior cruciate ligament reconstruction; n.s., non-significant

${ }^{a}$ One patient in the single ACL graft failure group underwent 2 subsequent surgical procedures, and 12 , 9,5 , and 1 patients in the multiple ACL graft failures group underwent 2, 3, 4, and 7 subsequent surgical procedures, respectively

${ }^{\mathrm{b}}$ One patient in the single ACL graft failure group and 6 patients in the multiple ACL graft failures group experienced two complications

*Statistically significant difference $(p<0.05)$ for primary ACL-R $[9,23,25]$, there are only few reports on the use of the quadriceps tendon as a graft for revision ACL-R [26, 27]. In one study, comparing ipsilateral quadriceps and contralateral hamstring tendon autografts for revision ACL-R, no difference with respect to PROs, instrumented laxity testing, and objective knee evaluation could be observed between the two graft options in 25 (quadriceps tendon) and 26 (hamstring tendon) patients, respectively [27]. However, using the ipsilateral quadriceps tendon had the decisive advantage of avoiding donor site morbidity on the unaffected contralateral limb. Another study showed consistent results with no difference in PROs and anterior-posterior laxity (KT-1000) between patients undergoing revision ACL-R using quadriceps tendon $(n=41)$ or hamstring tendon $(n=37)$ autografts [26]. Notably, after a mean follow-up period of 4.4 years, patients treated with quadriceps tendon revision ACL-R demonstrated significantly less residual rotatory knee laxity based on manual pivot-shift testing [26].

Multiple ACL graft failures are associated with an atraumatic injury mechanism with a gradual onset of recurrent instability $[5,12,16]$. This was confirmed by the present study, in which an atraumatic injury mechanism was significantly more often observed in the group with multiple compared to single ACL graft failures ( $45 \%$ vs. $22 \%$ ). This might be the result of persistent rotatory knee laxity, which would also explain the high prevalence of meniscal and cartilage lesions in multiple failed ACL-Rs $[1,5,12,13,15,16$, $28,29]$. Since concomitant meniscal and cartilage injuries are associated with the occurrence of $\mathrm{OA}$, it is not surprising that patients with ACL graft failures have significantly more OA compared to patients with an intact ACL graft at longterm follow-up [30]. Although, in this study, more patients with high-grade osteoarthritic changes (Kellgren-Lawrence 
Scale grade 3 and 4) were identified in the multiple compared to the single ACL graft failures group, no statistical significance was observed.

A higher (steeper) PTS has been associated with ACL injury and rotatory knee laxity, which is even more pronounced in patients with single or multiple ACL graft failures compared to patients with primary ACL injury [5, 13, $31,32]$. This is consistent with the findings of the present study, in which a significantly higher (steeper) medial PTS was observed in patients with multiple compared to single ACL graft failures $\left(12 \pm 3^{\circ}\right.$ vs. $\left.9 \pm 3^{\circ}\right)$. This observation confirms the multifactorial etiology of multiple ACL graft failures, which may lead to a vicious circle involving subsequent surgical procedures, complications, and reduced quality of life. The impact of multiple ACL graft failures on quality of life was demonstrated by significantly worse PROs compared to patients with single ACL graft failures. Given the low average level on the ACL-RSI scale for patients with multiple ACL graft failures, the psychological readiness to return to sport is severely compromised, causing many patients to change the type and level of physical activity [22].

An overall reoperation rate of $11 \%$ after revision ACL-R has been reported by the MARS group. Meniscal, revision ACL-R, and cartilage procedures accounted for $27 \%, 19 \%$, and $17 \%$, respectively, in the MARS cohort [33]. Interestingly, another study by the MARS group demonstrated that patients undergoing more than two revision ACL-Rs are 4.7 times more likely to require subsequent surgery compared to patients undergoing a first-time revision ACL-R [2]. This was also confirmed in the present study, in which patients with multiple ACL graft failures were more likely to undergo subsequent surgery and had significantly more subsequent surgical procedures than patients with a single ACL graft failure.

One limitation of the present study was the significantly longer follow-up period since the first revision ACL-R for patients with multiple compared to single ACL graft failures. Given that many patients already experienced multiple ACL graft failures at the time of first consultation, this was to be expected. Previous studies comparing the results of primary and multiple revision ACL-R may also be subjected to this bias, since the reported follow-up time is usually related to the last revision ACL-R [5, 12]. Comparing the follow-up period since the last revision ACL-R implicates a difference in the follow-up period since the first revision ACL-R, which is the most appropriate index procedure for comparing such patients. Patients in the single ACL graft failure group may experience subsequent ACL graft failures in the future. Therefore, a minimum of 12-month follow-up represents another limitation of this study. In addition, PROs were not available in all patients, which is associated with the retrospective design of this study.

\section{Conclusions}

In this study, multiple ACL graft failures were associated with worse PROs, higher (steeper) PTS, and the use of allografts compared to single ACL graft failures. During the first revision ACL reconstruction, it is recommended to avoid the use of allografts and to consider slope-reducing osteotomies to improve functional outcomes by reducing the risk of multiple ACL graft failures.

Acknowledgements Not applicable.

Author contributions All listed authors have contributed substantially to this work: PWW, NNW, and JDH collected data, performed statistical analysis, literature review, and primary manuscript preparation. VM and BPL assisted with interpretation of the results, initial drafting of the manuscript, as well as editing and final manuscript preparation. All authors read and approved the final manuscript.

Funding Open Access funding enabled and organized by Projekt DEAL.

\section{Compliance with ethical standards}

Ethical approval This study was approved by the Institutional Review Board of the University of Pittsburgh (No.: STUDY20050226).

Conflict of interest VM reports educational grants, consulting fees, and speaking fees from Smith \& Nephew plc, educational grants from Arthrex, is a board member of the International Society of Arthroscopy, Knee Surgery and Orthopaedic Sports Medicine (ISAKOS), and deputy editor-in-chief of Knee Surgery, Sports Traumatology, Arthroscopy (KSSTA). In addition, VM has a patent Quantified injury diagnostics-U.S. Patent No. 9,949,684, Issued on April 24, 2018 issued to University of Pittsburgh.

Informed consent Written informed consent was obtained from each patient who completed the questionnaire of this study.

Open Access This article is licensed under a Creative Commons Attribution 4.0 International License, which permits use, sharing, adaptation, distribution and reproduction in any medium or format, as long as you give appropriate credit to the original author(s) and the source, provide a link to the Creative Commons licence, and indicate if changes were made. The images or other third party material in this article are included in the article's Creative Commons licence, unless indicated otherwise in a credit line to the material. If material is not included in the article's Creative Commons licence and your intended use is not permitted by statutory regulation or exceeds the permitted use, you will need to obtain permission directly from the copyright holder. To view a copy of this licence, visit http://creativecommons.org/licenses/by/4.0/.

\section{References}

1. Alm L, Krause M, Frosch KH, Akoto R (2020) Preoperative medial knee instability is an underestimated risk factor for failure of revision ACL reconstruction. Knee Surg Sports Traumatol Arthrosc. https://doi.org/10.1007/s00167-020-06133-y 
2. MARS Group (2014) Effect of graft choice on the outcome of revision anterior cruciate ligament reconstruction in the Multicenter ACL Revision Study (MARS) Cohort. Am J Sports Med 42:2301-2310

3. Mohan R, Webster KE, Johnson NR, Stuart MJ, Hewett TE, Krych $\mathrm{AJ}$ (2018) Clinical outcomes in revision anterior cruciate ligament reconstruction: a meta-analysis. Arthroscopy 34:289-300

4. Ouillette R, Edmonds E, Chambers H, Bastrom T, Pennock A (2019) Outcomes of revision anterior cruciate ligament surgery in adolescents. Am J Sports Med 47:1346-1352

5. Yoon KH, Kim JH, Kwon YB, Kim EJ, Kim SG (2020) Rerevision anterior cruciate ligament reconstruction showed more laxity than revision anterior cruciate ligament reconstruction at a minimum 2-year follow-up. Knee Surg Sports Traumatol Arthrosc 28:1909-1918

6. Getgood AMJ, Bryant DM, Litchfield R, Heard M, McCormack RG, Rezansoff A et al (2020) Lateral extra-articular tenodesis reduces failure of hamstring tendon autograft anterior cruciate ligament reconstruction: 2-year outcomes from the STABILITY Study Randomized Clinical Trial. Am J Sports Med 48:285-297

7. Gifstad T, Foss OA, Engebretsen L, Lind M, Forssblad M, Albrektsen $\mathrm{G}$ et al (2014) Lower risk of revision with patellar tendon autografts compared with hamstring autografts: a registry study based on 45,998 primary ACL reconstructions in Scandinavia. Am J Sports Med 42:2319-2328

8. Persson A, Fjeldsgaard K, Gjertsen JE, Kjellsen AB, Engebretsen L, Hole RM et al (2014) Increased risk of revision with hamstring tendon grafts compared with patellar tendon grafts after anterior cruciate ligament reconstruction: a study of 12,643 patients from the Norwegian Cruciate Ligament Registry, 2004-2012. Am J Sports Med 42:285-291

9. Runer A, Csapo R, Hepperger C, Herbort M, Hoser C, Fink C (2020) Anterior cruciate ligament reconstructions with quadriceps tendon autograft result in lower graft rupture rates but similar patient-reported outcomes as compared with hamstring tendon autograft: a comparison of 875 patients. Am J Sports Med 48:2195-2204

10. Wright RW, Dunn WR, Amendola A, Andrish JT, Bergfeld J, Kaeding CC et al (2007) Risk of tearing the intact anterior cruciate ligament in the contralateral knee and rupturing the anterior cruciate ligament graft during the first 2 years after anterior cruciate ligament reconstruction: a prospective MOON cohort study. Am J Sports Med 35:1131-1134

11. Wright RW, Magnussen RA, Dunn WR, Spindler KP (2011) Ipsilateral graft and contralateral ACL rupture at five years or more following ACL reconstruction: a systematic review. J Bone Joint Surg Am 93:1159-1165

12. Chen JL, Allen CR, Stephens TE, Haas AK, Huston LJ, Wright RW et al (2013) Differences in mechanisms of failure, intraoperative findings, and surgical characteristics between single- and multiple-revision ACL reconstructions: a MARS cohort study. Am J Sports Med 41:1571-1578

13. Grassi A, Macchiarola L, Urrizola Barrientos F, Zicaro JP, Costa Paz M, Adravanti P et al (2019) Steep posterior tibial slope, anterior tibial subluxation, deep posterior lateral femoral condyle, and meniscal deficiency are common findings in multiple anterior cruciate ligament failures: an MRI Case-Control Study. Am J Sports Med 47:285-295

14. Cooper DE, Dunn WR, Huston LJ, Haas AK, Spindler KP et al (2018) Physiologic preoperative knee hyperextension is a predictor of failure in an anterior cruciate ligament revision cohort: a report from the MARS Group. Am J Sports Med 46:2836-2841

15. Wegrzyn J, Chouteau J, Philippot R, Fessy MH, Moyen B (2009) Repeat revision of anterior cruciate ligament reconstruction: a retrospective review of management and outcome of 10 patients with an average 3-year follow-up. Am J Sports Med 37:776-785
16. Griffith TB, Allen BJ, Levy BA, Stuart MJ, Dahm DL (2013) Outcomes of repeat revision anterior cruciate ligament reconstruction. Am J Sports Med 41:1296-1301

17. Allen CR, Anderson AF, Cooper DE, DeBerardino TM, Dunn WR et al (2017) Surgical predictors of clinical outcomes after revision anterior cruciate ligament reconstruction. Am J Sports Med 45:2586-2594

18. Napier RJ, Garcia E, Devitt BM, Feller JA, Webster KE (2019) Increased radiographic posterior Tibial slope is associated with subsequent injury following revision anterior cruciate ligament reconstruction. Orthop J Sports Med 7:2325967119879373

19. Pfeiffer TR, Burnham JM, Hughes JD, Kanakamedala AC, Herbst E, Popchak A et al (2018) An increased lateral femoral condyle ratio is a risk factor for anterior cruciate ligament injury. $\mathrm{J}$ Bone Joint Surg Am 100:857-864

20. Pfeiffer TR, Burnham JM, Kanakamedala AC, Hughes JD, Zlotnicki J, Popchak A et al (2019) Distal femur morphology affects rotatory knee instability in patients with anterior cruciate ligament ruptures. Knee Surg Sports Traumatol Arthrosc 27:1514-1519

21. Weinberg DS, Williamson DF, Gebhart JJ, Knapik DM, Voos JE (2017) Differences in medial and lateral posterior Tibial slope: an osteological review of 1090 tibiae comparing age, sex, and race. Am J Sports Med 45:106-113

22. Langford JL, Webster KE, Feller JA (2009) A prospective longitudinal study to assess psychological changes following anterior cruciate ligament reconstruction surgery. Br J Sports Med 43:377-381

23. Runer A, Wierer G, Herbst E, Hepperger C, Herbort M, Gföller $P$ et al (2018) There is no difference between quadriceps- and hamstring tendon autografts in primary anterior cruciate ligament reconstruction: a 2-year patient-reported outcome study. Knee Surg Sports Traumatol Arthrosc 26:605-614

24. Sheean AJ, Musahl V, Slone HS, Xerogeanes JW, Milinkovic D, Fink C et al (2018) Quadriceps tendon autograft for arthroscopic knee ligament reconstruction: use it now, use it often. Br J Sports Med 52:698-701

25. Cavaignac E, Coulin B, Tscholl P, Nik Mohd Fatmy N, Duthon V, Menetrey J (2017) Is quadriceps tendon autograft a better choice than hamstring autograft for anterior cruciate ligament reconstruction? A comparative study with a mean follow-up of 3.6 years. Am J Sports Med 45:1326-1332

26. Barié A, Ehmann Y, Jaber A, Huber J, Streich NA (2019) Revision ACL reconstruction using quadriceps or hamstring autografts leads to similar results after 4 years: good objective stability but low rate of return to pre-injury sport level. Knee Surg Sports Traumatol Arthrosc 27:3527-3535

27. Häner M, Bierke S, Petersen W (2016) Anterior cruciate ligament revision surgery: ipsilateral quadriceps versus contralateral semitendinosus-gracilis autografts. Arthroscopy 32:2308-2317

28. Musahl V, Citak M, O'Loughlin PF, Choi D, Bedi A, Pearle AD (2010) The effect of medial versus lateral meniscectomy on the stability of the anterior cruciate ligament-deficient knee. Am J Sports Med 38:1591-1597

29. Musahl V, Rahnemai-Azar AA, Costello J, Arner JW, Fu FH, Hoshino Y et al (2016) The influence of meniscal and anterolateral capsular injury on knee laxity in patients with anterior cruciate ligament injuries. Am J Sports Med 44:3126-3131

30. Söderman T, Wretling ML, Hänni M, Mikkelsen C, Johnson RJ, Werner S et al (2020) Higher frequency of osteoarthritis in patients with ACL graft rupture than in those with intact ACL grafts 30 years after reconstruction. Knee Surg Sports Traumatol Arthrosc 28:2139-2146

31. Grassi A, Signorelli C, Urrizola F, Macchiarola L, Raggi F, Mosca $M$ et al (2019) Patients with failed anterior cruciate ligament reconstruction have an increased posterior lateral tibial plateau slope: a case-controlled study. Arthroscopy 35:1172-1182 
32. Rahnemai-Azar AA, Abebe ES, Johnson P, Labrum J, Fu FH, Irrgang JJ et al (2017) Increased lateral tibial slope predicts highgrade rotatory knee laxity pre-operatively in ACL reconstruction. Knee Surg Sports Traumatol Arthrosc 25:1170-1176

33. Ding DY, Zhang AL, Allen CR, Anderson AF, Cooper DE et al (2017) Subsequent surgery after revision anterior cruciate ligament reconstruction: rates and risk factors from a multicenter cohort. Am J Sports Med 45:2068-2076
Publisher's Note Springer Nature remains neutral with regard to jurisdictional claims in published maps and institutional affiliations. 